\title{
Experimental Studies on Laminar Jet Methane-Air Premixed Flame at Atmospheric Condition
}

\author{
Jahidul Haque Chaudhuri \\ Direct Ph.D., Department of Mechanical Engineering, Indian Institute of Technology Madras, Chennai, 600036, India \\ DOI: https://dx.doi.org/10.51244/IJRSI.2020.7801
}

\begin{abstract}
Flame can be defined in which rapid chemical reaction take place and often emitting light. In other words, flame is a self-sustainable localized combustion zone that moves at a certain velocity into the fuel-air mixture. This is also known as a combustion wave since it moves and there is a sudden change in properties like temperature and mass fraction of constituent species. Combustion can occur in both in flame and non-flame method. Flame mode further classified premixed flame and nonpremixed fame. A premixed flame can be stabilized between two limiting values of gas flow rate. First limiting value of gas flow rate is, for a certain maximum value of gas flow rate flame get detached from the burner and flame go away from the domain of interest. This phenomenon is called blow off or liftoff the flame. During liftoff condition flame may be stabilized at some distance from the port. Due to liftoff, several problems create like escape or loss of unburnt gasses, poor heat transfer, noisy, incomplete combustion, and tough to accurately control the position of flame. And second, the limiting value of gas flow rate is, when the flow rate of the gas falls below a certain minimum value, the flame is not capable of anchoring itself to the burner rim and moves into the burner, this phenomenon is called flashback. Similar to liftoff conditions due to flashbacks create several problems as it can lead to an explosion. So data off flashback and blow off is very important to design any combustion system. In this present study focusing on finding stability range of methaneair experimentally by investigating flashback and blow off covering a wide range of fuel-air ratio from lean to reach mixture. And also observe how cone angle and flame height changes with changing air-fuel flow rate, using flame cone angle flame propagation speed can measure and compare at which condition (lean, rich, stoichiometric) its maximum.
\end{abstract}

Keywords- Methane-air mixer, Stability-limit, Premixed-flame, laminar, lean, Rich, Stoichiometric.

\section{INTRODUCTION}

$\mathrm{L}$ aminar flame speed is one of the important parameter of the flame, it's used in various area like as a basic data to examine the flame instability or as a validation data to refine chemical kinetics model. The study of earlier works reveals that a great deal of work has been carried out in areas of stability of premixed hydrocarbon flame. Stability of propane air flames determined by flashback and blow off investigated by chen and Churchill [1]. Mishra [2] experimentally investigated the stability range of $\mathrm{CNC}$-air premixed flame. He also observed that the flashback limit could be enhanced with an increase in burner diameter. Hydrogen-air premixed flame stability studied under different inlet velocity and wall thermal conductivity in annular micro combustor by Jejurkar et al. [3]. Khandelwal [4] investigated the flame stability limit of the premixed methane-air mixture in a backward step microscale combustor. On a bluff-body burner, Chen et al. [5] investigated the mechanism of flame Lift-off and stabilization of non-premixed flames. Leung and wierzba [6] observed how stability (non-premixed) effected by hydrogen addition on biogas flame. They are also investigated how jet diameter impacts flame stability.Hu et al. [7] investigated laminar premixed flame methane-air at various pressure by numerically and experimentally. Stability of methane-oxygen premixed flame find experimentally harris er al.[8]. Similarly, Wohl et al. [9] studied the stability of butane-air flame. Schefer [10] show how hydrogen addition improved lean film stability. Jerzak and kuznia [11] experimentally investigated the effect of swirl number and oxygen, carbon dioxide content in natural gas combustion. In generic swirl burner, Syred et al. [12] studied the effect of inlet-outlet configuration on flashback and blow-off (premixed combustion of methane and hydrogen content fuel). Krikunova [13] studied how premixed methane-air flame behave at alternate gravity. Yuasa et al [14] investigated the combustion characteristic of the ultra-micro combustor with premixes flame. Mishra and Kannan [15] experimentally work on swirl burner to investigate lean premixed combustion. This article focused on finding experimentally the stability region of methane-air premixed combustion on Bunsen burner (burner dia. $11.2 \mathrm{~mm}$ ) at three different conditions (lean, Rich, Stoichiometric).And also analysis the picture, which get experimentally.

\section{EXPERIMENTAL METHOD}

I. Connect all filter, moisture collector, Mass flow controller as shown in fig 1.

II. The required $\mathrm{CH}_{4}$ for this experiment is taken from the commercially available cylinder and air from the highly pressurized tank

III. Air \& fuel $\left(\mathrm{CH}_{4}\right)$ mixed in a burner.

IV. The flow rate is controlled by the mass flow controller. By changing MFC set value get the different flame structures.

V. Blow off is determined by increasing the total gas flow rate in small increments until flame lift-off from burner port.

VI. Similarly, the flashback is determined by decreasing the gas flow rate.

VII. With the help of a camera take picture of all flame.

VIII. Import this photo in imagej software and find cone angle. 
IX. With the help of cone angle, find flame speed.

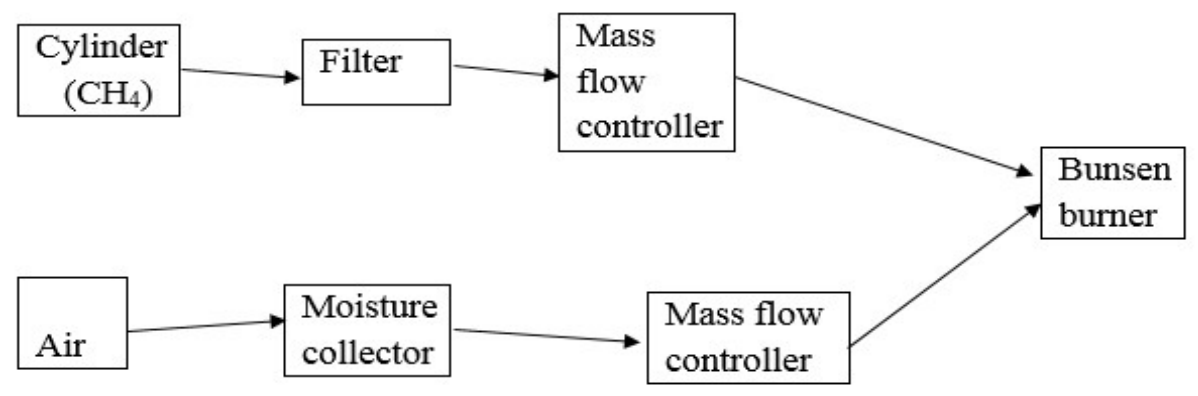

Fig.1 Experimental setup

\section{EXPERIMENTAL DATA}

a. Burner inner diameter $=11.2 \mathrm{~mm}$

$$
\text { Area }=98.52 \mathrm{~mm}^{2}=0.00009852 \mathrm{~m}^{2}
$$

Table 2.1 (Identifying flashback \& blow off at Stoichiometric condition)

\begin{tabular}{|c|c|c|c|c|c|c|}
\hline SL. No. & $\begin{array}{l}\text { Fuel flow } \\
\text { rate ( slpm ) }\end{array}$ & $\begin{array}{l}\text { Oxidizer flow } \\
\text { rate (slpm ) }\end{array}$ & $\begin{array}{c}\text { Flashback/ } \\
\text { blow off }\end{array}$ & $\begin{array}{c}\text { Avg. Velocity } \\
(\mathrm{m} / \mathrm{s})\end{array}$ & $\mathrm{A} / \mathrm{F}$ & $\phi$ \\
\hline 1 & 0.955 & 9.09 & - & 1.7 & 9.52 & 1 \\
\hline 2 & 0.842 & 8.02 & - & 1.5 & 9.52 & 1 \\
\hline 3 & 0.786 & 7.48 & - & 1.4 & 9.52 & 1 \\
\hline 4 & 0.618 & 5.88 & - & 1.1 & 9.52 & 1 \\
\hline 5 & 0.5619 & 5.34 & - & 1 & 9.52 & 1 \\
\hline 6 & 0.449 & 4.27 & - & 0.8 & 9.52 & 1 \\
\hline 7 & 0.337 & 3.20 & - & 0.6 & 9.52 & 1 \\
\hline 8 & 0.225 & 2.14 & Flash back & 0.4 & 9.52 & 1 \\
\hline 9 & 0.168 & 1.60 & Flash back & 0.3 & 9.52 & 1 \\
\hline
\end{tabular}

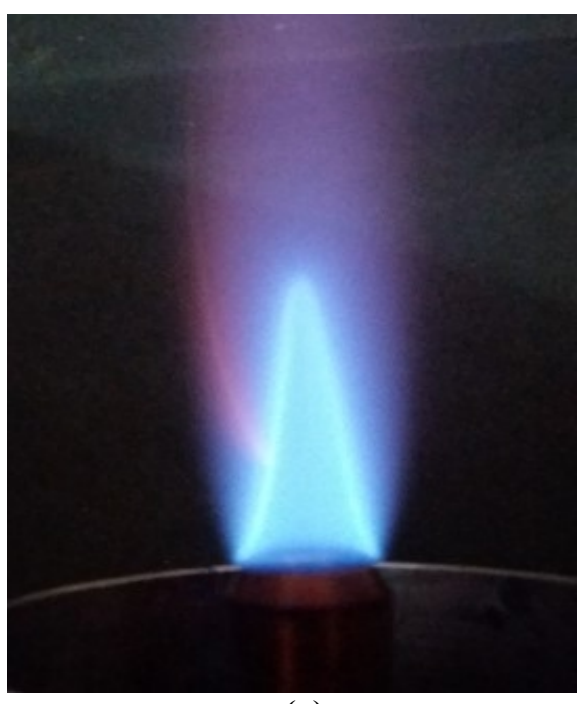

(a)

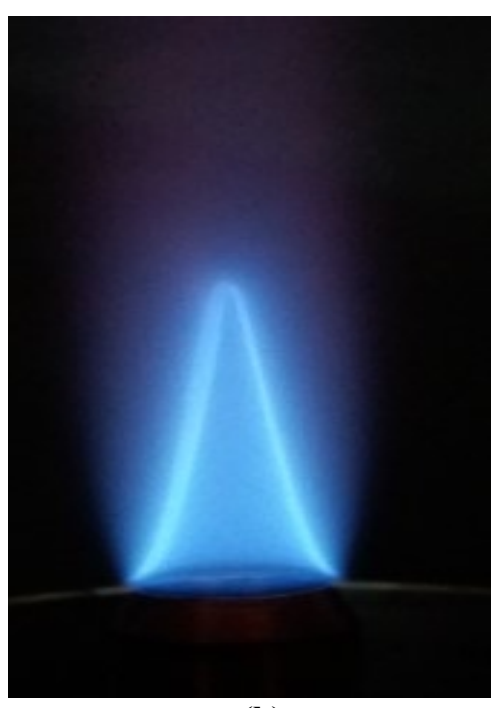

(b)

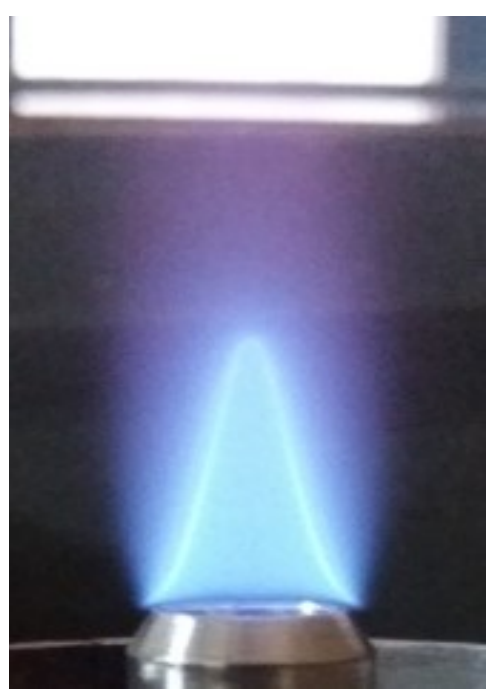

(c) 


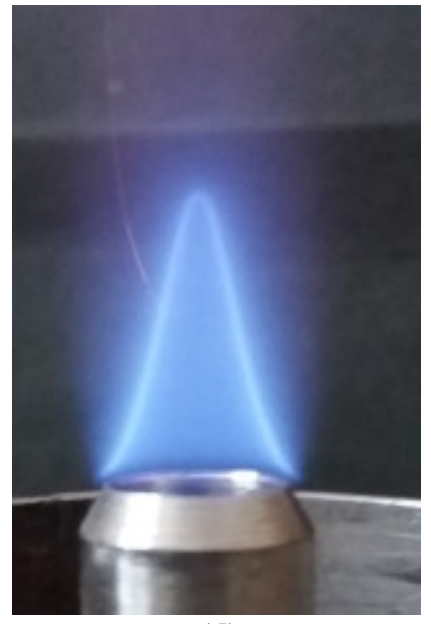

(d)

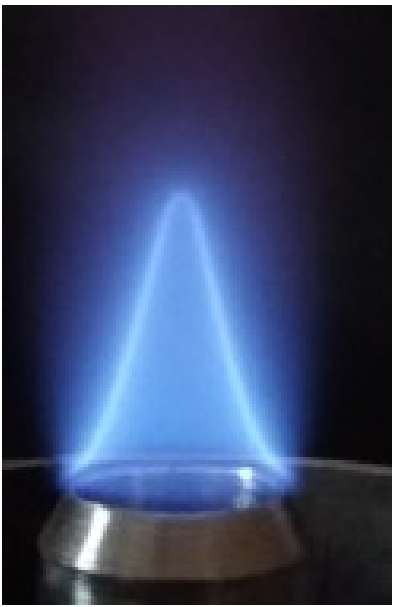

(e)

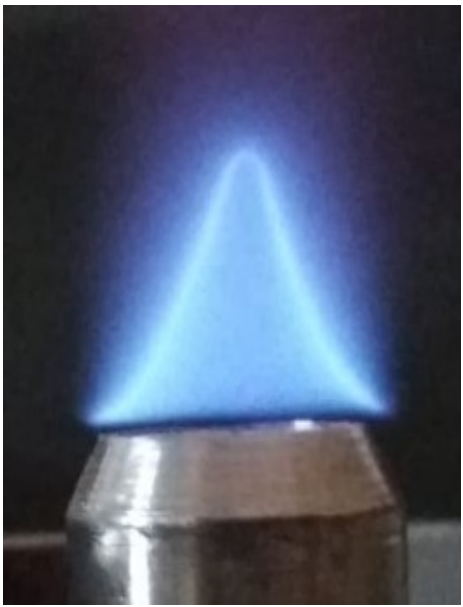

(f)

Fig. 1.2: Flame structure for mixture velocity of (a) $1.7 \mathrm{~m} / \mathrm{s}$, (b) $1.5 \mathrm{~m} / \mathrm{s}$, (c) $1.1 \mathrm{~m} / \mathrm{s},(\mathrm{d}) 1 \mathrm{~m} / \mathrm{s},(\mathrm{e}) 0.8 \mathrm{~m} / \mathrm{s},(\mathrm{f}) 0.6 \mathrm{~m} / \mathrm{s}$.

b. Burner inner diameter $=11.2 \mathrm{~mm}$

Area $=98.52 \mathrm{~mm}^{2}=0.00009852 \mathrm{~m}^{2}$

$\Phi=1.1$

Table 2.2 (Identifying flashback \& blow off at rich condition)

\begin{tabular}{|c|c|c|c|c|c|c|}
\hline S.No & $\begin{array}{l}\text { Fuel flow } \\
\text { rate }(\mathrm{Lpm})\end{array}$ & $\begin{array}{l}\text { Oxidizer flow } \\
\text { rate }(\mathrm{Lpm})\end{array}$ & $\begin{array}{c}\text { Flashback/ } \\
\text { blow off }\end{array}$ & $\begin{array}{l}\text { Avg. Velocity } \\
(\mathrm{m} / \mathrm{s})\end{array}$ & $\mathrm{A} / \mathrm{F}$ & $\phi$ \\
\hline 1 & 0.918 & 7.95 & - & 1.5 & 8.65 & 1.1 \\
\hline 2 & 0.857 & 7.42 & - & 1.4 & 8.65 & 1.1 \\
\hline 3 & 0.674 & 5.83 & - & 1.1 & 8.65 & 1.1 \\
\hline 4 & 0.612 & 5.29 & - & 1 & 8.65 & 1.1 \\
\hline 5 & 0.490 & 4.24 & - & 0.8 & 8.66 & 1.1 \\
\hline 6 & 0.428 & 3.71 & - & 0.7 & 8.65 & 1.1 \\
\hline 7 & 0.367 & 3.18 & Flash back & 0.6 & 8.65 & 1.1 \\
\hline
\end{tabular}

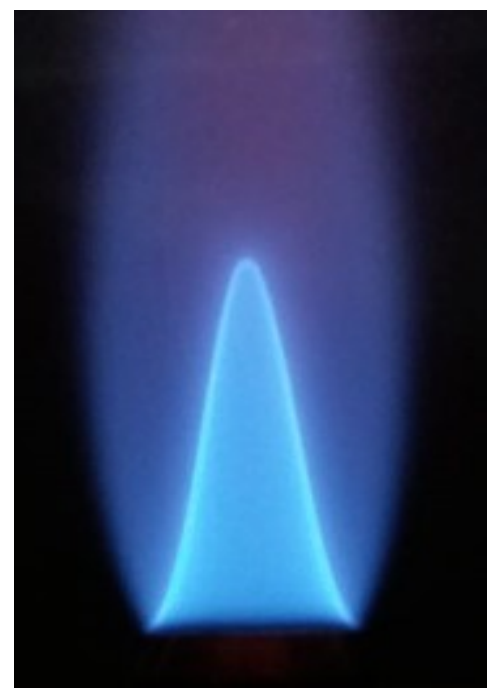

(a)

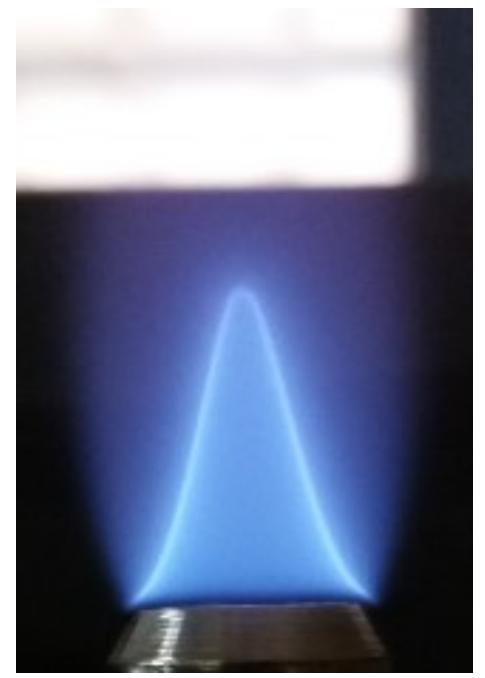

(b)

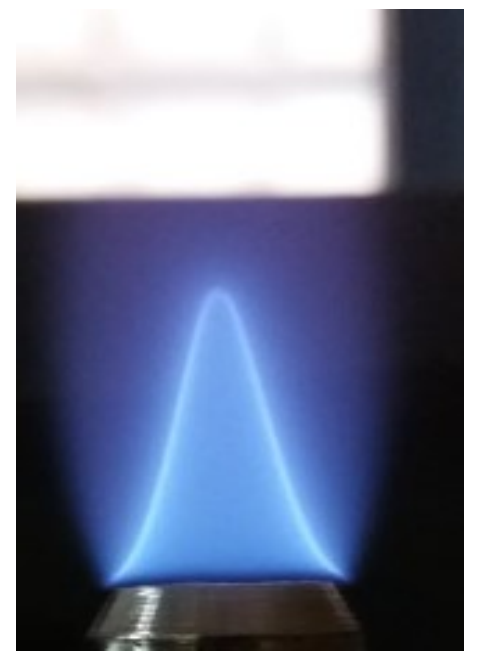

(c) 


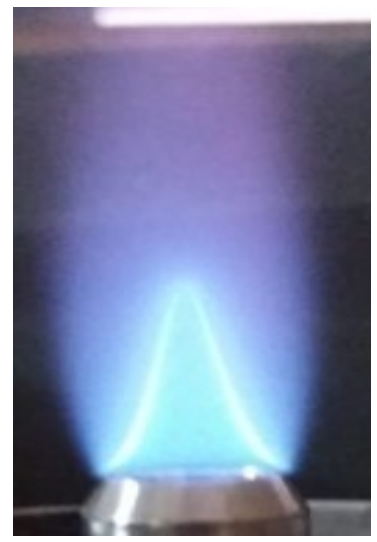

(d)

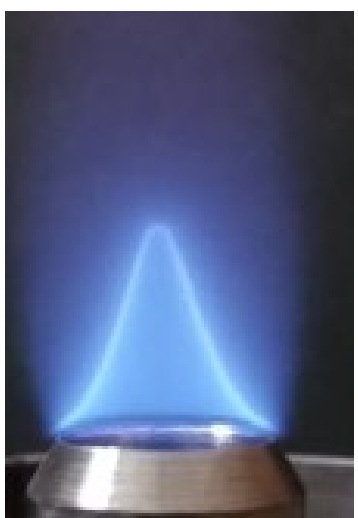

(e)

Fig. 1.3: Flame structure for mixture velocity of (a) $1.5 \mathrm{~m} / \mathrm{s}$, (b) $1.4 \mathrm{~m} / \mathrm{s}$, , c) $1.1 \mathrm{~m} / \mathrm{s}$, (d) $1 \mathrm{~m} / \mathrm{s}$, , (e) $0.8 \mathrm{~m} / \mathrm{s}$,

c. Burner inner diameter $=11.2 \mathrm{~mm}$

Area $=98.52 \mathrm{~mm}^{2}=0.00009852 \mathrm{~m}^{2}$

$\Phi=1.2$

Table 2.3 (Identifying flashback \& blow off at rich condition)

\begin{tabular}{|c|c|c|c|c|c|c|}
\hline S.No & $\begin{array}{c}\text { Fuel flow } \\
\text { rate (Lpm) }\end{array}$ & $\begin{array}{c}\text { Oxidizer flow } \\
\text { rate (Lpm) }\end{array}$ & $\begin{array}{c}\text { Flashback/ } \\
\text { blow off }\end{array}$ & $\begin{array}{c}\text { Avg. Velocity } \\
(\mathrm{m} / \mathrm{s})\end{array}$ & $\mathrm{A} / \mathrm{F}$ & $\phi$ \\
\hline 1 & 0.994 & 7.84 & - & 1.5 & 7.93 & 1.2 \\
\hline 2 & 0.927 & 7.35 & - & 1.4 & 7.93 & 1.2 \\
\hline 3 & 0.861 & 6.82 & - & 1.3 & 7.93 & 1.2 \\
\hline 4 & 0.728 & 5.77 & - & 1.1 & 7.93 & 1.2 \\
\hline 5 & 0.662 & 5.25 & - & 1 & 7.93 & 1.2 \\
\hline 6 & 0.529 & 4.19 & - & 0.8 & 7.94 & 1.2 \\
\hline 7 & 0.463 & 3.67 & - & 0.7 & 7.92 & 1.2 \\
\hline 8 & 0.397 & 3.15 & flashback & 0.6 & 7.93 & 1.2 \\
\hline 9 & 0.265 & 2.09 & flashback & 0.4 & 7.93 & 1.2 \\
\hline
\end{tabular}

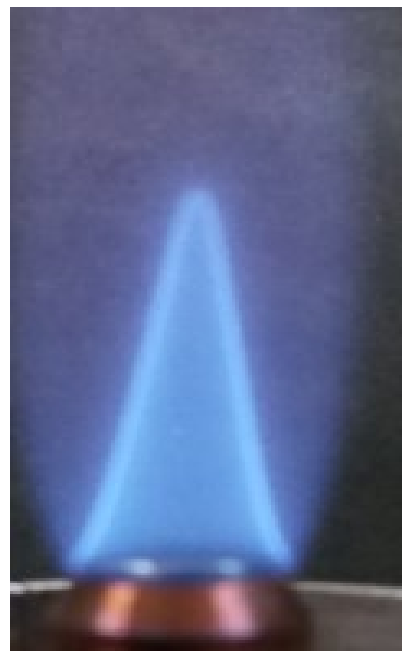

(a)

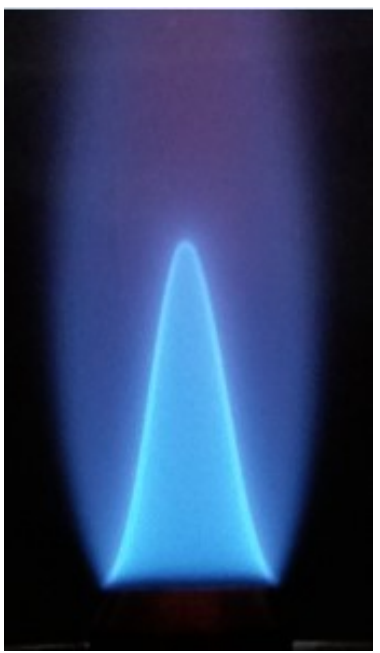

(b)

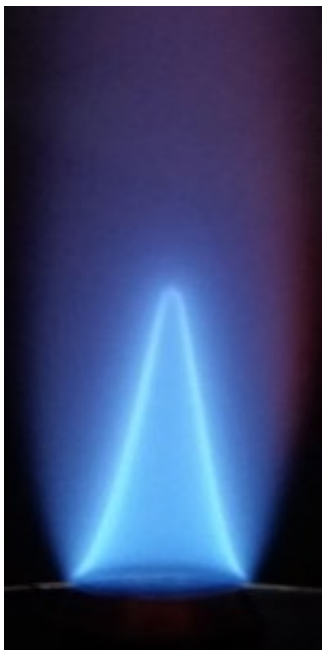

(c)

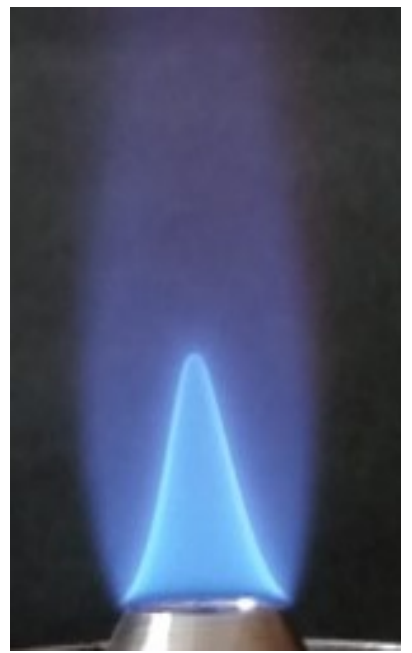

(d)

Fig.1.4: Flame structure for mixture velocity of (a) $1.5 \mathrm{~m} / \mathrm{s}$, (b) $1.4 \mathrm{~m} / \mathrm{s}$, (c) $1.1 \mathrm{~m} / \mathrm{s}$, (d) $1 \mathrm{~m} / \mathrm{s}$. 
d. Burner inner diameter $=11.2 \mathrm{~mm}$

Area $=98.52 \mathrm{~mm}^{2}=0.00009852 \mathrm{~m}^{2}$

$\Phi=1.3$

Table 2.4. (Identifying flashback \& blow off at rich condition)

\begin{tabular}{|c|c|c|c|c|c|c|}
\hline S.No & $\begin{array}{l}\text { Fuel flow } \\
\text { rate (Lpm) }\end{array}$ & $\begin{array}{c}\text { Oxidizer flow } \\
\text { rate (Lpm) }\end{array}$ & $\begin{array}{c}\text { Flashback/ } \\
\text { blow off }\end{array}$ & $\begin{array}{c}\text { Avg. Velocity } \\
(\mathrm{m} / \mathrm{s})\end{array}$ & $\mathrm{A} / \mathrm{F}$ & $\phi$ \\
\hline 1 & 0.994 & 7.28 & - & 1.4 & 7.32 & 1.3 \\
\hline 2 & 0.923 & 6.76 & - & 1.3 & 7.32 & 1.3 \\
\hline 3 & 0.782 & 5.72 & - & 1.1 & 7.32 & 1.3 \\
\hline 4 & 0.710 & 5.20 & - & 1 & 7.32 & 1.3 \\
\hline 5 & 0.568 & 4.16 & - & 0.8 & 7.32 & 1.3 \\
\hline 6 & 0.497 & 3.64 & - & 0.7 & 7.32 & 1.3 \\
\hline 7 & 0.426 & 3.12 & - & 0.6 & 7.32 & 1.3 \\
\hline 8 & 0.2842 & 2.08 & - & 0.4 & 7.32 & 1.3 \\
\hline 9 & 0.213 & 1.56 & flashback & 0.3 & 7.32 & 1.3 \\
\hline
\end{tabular}

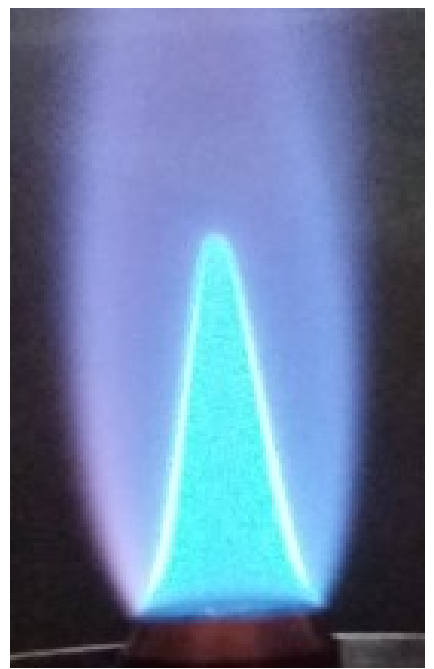

(a)

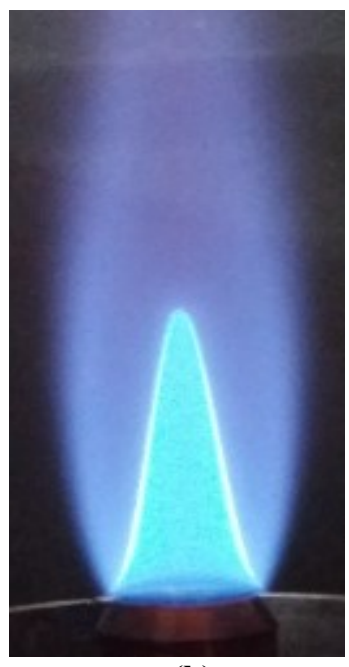

(b)

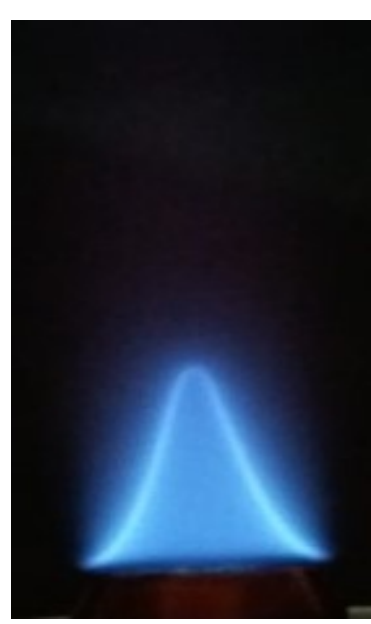

(e)

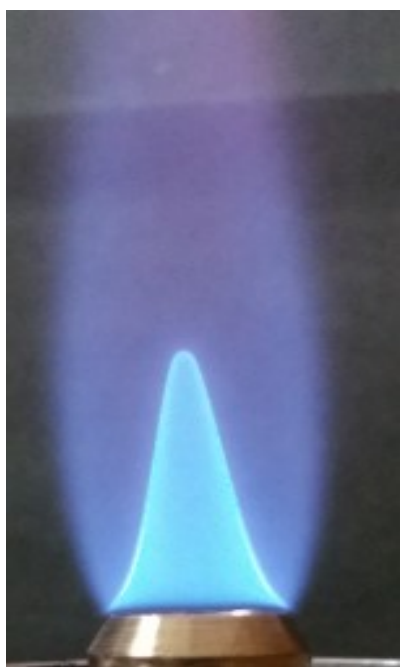

(c)

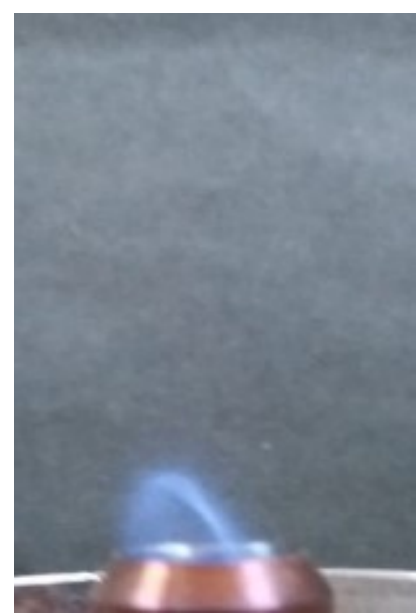

(f)

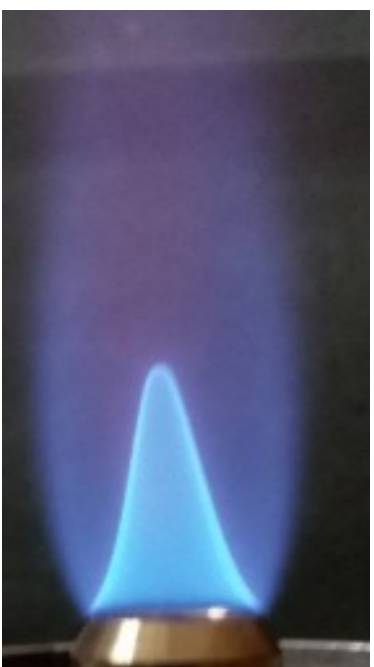

(d)

Fig.1.5: Flame structure for mixture velocity of (a) $1.4 \mathrm{~m} / \mathrm{s}$, (b) $1.3 \mathrm{~m} / \mathrm{s},$, (c) $1.1 \mathrm{~m} / \mathrm{s}$, , (d) $1 \mathrm{~m} / \mathrm{s}$, (e) $0.6 \mathrm{~m} / \mathrm{s},$, (f) $0.3 \mathrm{~m} / \mathrm{s}$. 
e. Burner inner diameter $=11.2 \mathrm{~m}$

Area $=98.52 \mathrm{~mm}^{2}=0.00009852 \mathrm{~m}^{2}$

$\Phi=0.9$

Table 2.5. (Identifying flashback \& blow off at lean condition)

\begin{tabular}{|c|c|c|c|c|c|c|}
\hline S.No & $\begin{array}{l}\text { Fuel flow } \\
\text { rate }(\mathrm{Lpm})\end{array}$ & $\begin{array}{c}\text { Oxidizer flow } \\
\text { rate }(\mathrm{Lpm})\end{array}$ & $\begin{array}{c}\begin{array}{c}\text { Flashback/ } \\
\text { blow off }\end{array} \\
\end{array}$ & $\begin{array}{l}\text { Avg. Velocity } \\
(\mathrm{m} / \mathrm{s})\end{array}$ & $\mathrm{A} / \mathrm{F}$ & $\phi$ \\
\hline 2 & 0.511 & 5.40 & & 1.0 & 10.57 & 0.9 \\
\hline 3 & 0.459 & 4.86 & & 0.9 & 10.57 & 0.9 \\
\hline 4 & 0.409 & 4.32 & & 0.8 & 10.57 & 0.9 \\
\hline 5 & 0.357 & 3.78 & & 0.7 & 10.57 & 0.9 \\
\hline 7 & 0.204 & 2.16 & flash back & 0.4 & 10.58 & 0.9 \\
\hline 8 & 0.153 & 1.62 & Flash back & 0.3 & 10.57 & 0.9 \\
\hline
\end{tabular}

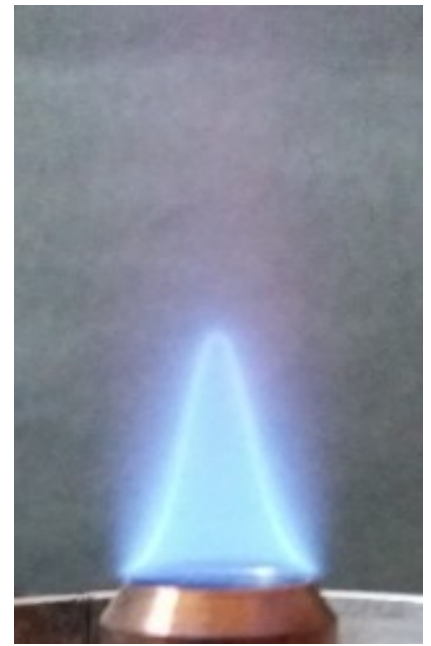

(a)

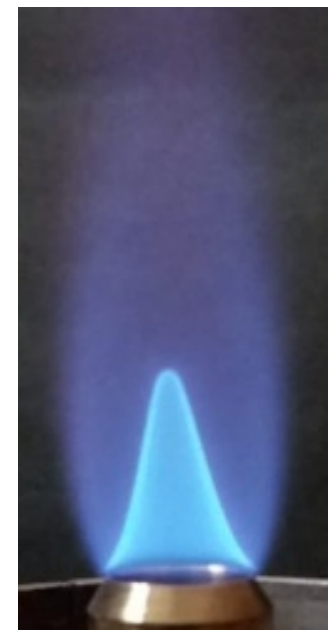

(b)

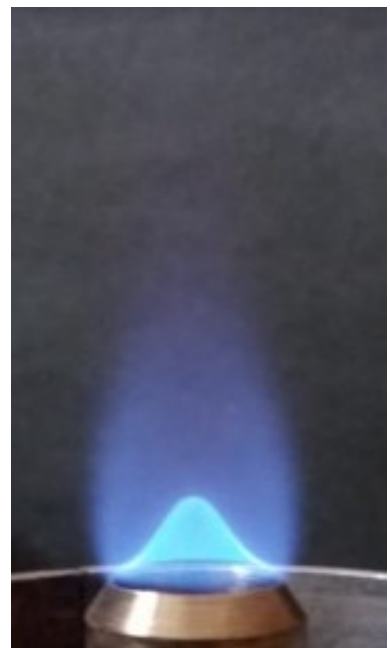

(c)

Fig.1.6: Flame structure for mixture velocity of (a) $1 \mathrm{~m} / \mathrm{s}$, (b) $0.9 \mathrm{~m} / \mathrm{s}$, (c) $0.7 \mathrm{~m} / \mathrm{s}$.

f. Burner inner diameter $=11.2 \mathrm{~mm}$

Area $=98.52 \mathrm{~mm}^{2}=0.00009852 \mathrm{~m}^{2}$

$\Phi=0.8($ Lean Mixture)

Table 2.6. Identifying flashback \& blow off at lean condition)

\begin{tabular}{|c|c|c|c|c|c|c|}
\hline S.No & $\begin{array}{l}\text { Fuel flow } \\
\text { rate }(\mathrm{Lpm})\end{array}$ & $\begin{array}{c}\text { Oxidizer flow } \\
\text { rate (Lpm) }\end{array}$ & $\begin{array}{c}\begin{array}{c}\text { Flashback/ } \\
\text { blow off }\end{array} \\
\end{array}$ & $\begin{array}{l}\text { Avg. Velocity } \\
(\mathrm{m} / \mathrm{s})\end{array}$ & $\mathrm{A} / \mathrm{F}$ & $\phi$ \\
\hline 1 & 0.504 & 5.99 & & 1.1 & 11.9 & 0.8 \\
\hline 2 & 0.458 & 5.45 & & 1 & 11.9 & 0.8 \\
\hline 3 & 0.366 & 4.36 & & 0.8 & 11.9 & 0.8 \\
\hline 4 & 0.321 & 3.81 & & 0.7 & 11.9 & 0.8 \\
\hline 5 & 0.274 & 3.27 & & 0.6 & 11.9 & 0.8 \\
\hline 6 & 0.229 & 2.73 & & 0.5 & 11.9 & 0.8 \\
\hline 7 & 0.183 & 2.18 & Flash back & 0.4 & 11.9 & 0.8 \\
\hline 8 & 0.137 & 1.64 & Flash back & 0.3 & 11.9 & 0.8 \\
\hline
\end{tabular}




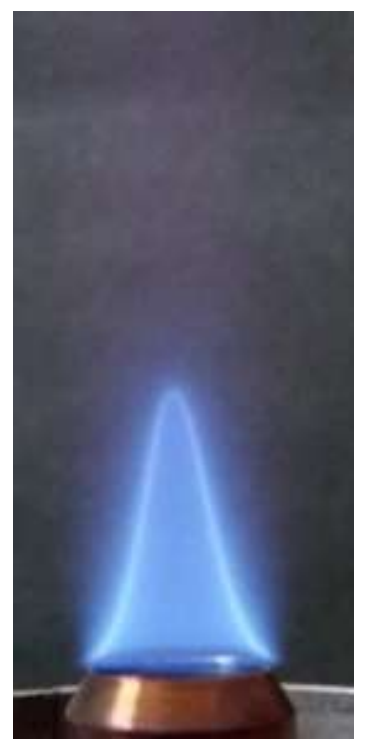

(a)

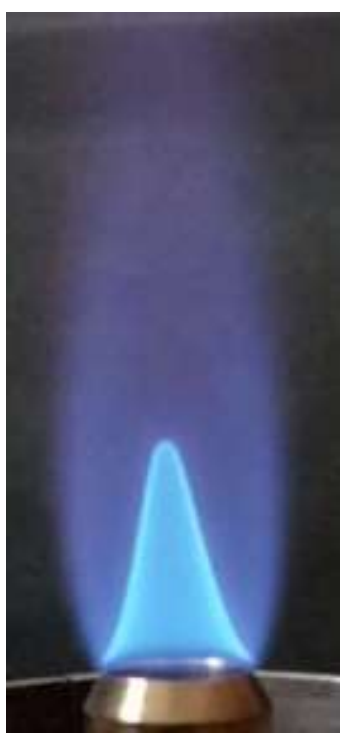

(b)

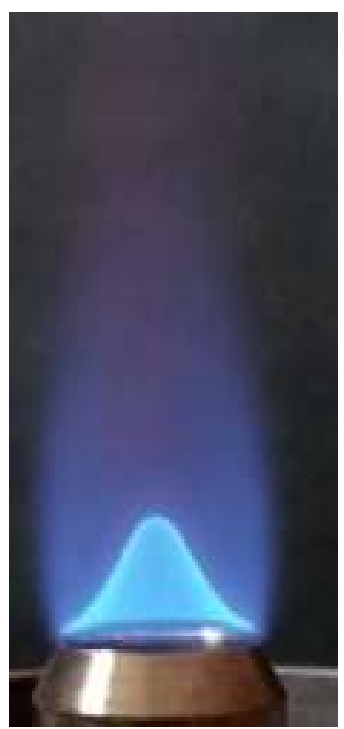

(c)

Fig. 1.7: Flame structure for mixture velocity of (a) $1 \mathrm{~m} / \mathrm{s}$, (b) $0.8 \mathrm{~m} / \mathrm{s}$, (c) $0.6 \mathrm{~m} / \mathrm{s}$.

\section{OBSERVATION}

Table 2.1 to 2.6 shown the experimental results. From this result stability region can be identified at three conditions (lean, rich, Stoichiometric) and at various air-fuel flow rates. And figure 1.2 to 1.7 shown flame structure at various mixture velocity. Table 3 shows a comparison between flashback and flame propagation speed. Figure 4 graphically represents the variation of flame speed with equivalence ratio.

Table 3 (comparison between flashback and flame propagation speed)

\begin{tabular}{|c|c|c|c|c|c|}
\hline$\phi$ & $\begin{array}{c}\mathrm{U} \\
\text { (unburnt } \\
\text { Mixture speed)m/s }\end{array}$ & $\begin{array}{c}\mathrm{S}_{\mathrm{L}}=\mathrm{u}^{*} \sin \alpha \\
\text { (flame propagation } \\
\text { speed)m/s }\end{array}$ & $\begin{array}{c}\text { Flash } \\
\text { Back } \\
\mathrm{m} / \mathrm{s}\end{array}$ & $\begin{array}{c}\text { Blow off } \\
\mathrm{m} / \mathrm{s}\end{array}$ & $\begin{array}{c}\alpha^{0} \\
\text { (Cone angle of flame) }\end{array}$ \\
\hline 1 & 1 & 0.29 & 0.4 & $>1.7$ & 32.856 \\
\hline 1.1 & 1 & 0.315 & 0.4 & $>1.7$ & 36.774 \\
\hline 1.2 & 1 & 0.283 & 0.6 & $>1.5$ & 32.883 \\
\hline 1.3 & 1 & 0.243 & 0.6 & $>1.5$ & 28.181 \\
\hline 0.9 & 1 & 0.275 & 0.3 & $>1.4$ & 32.001 \\
\hline 0.8 & 1 & 0.26 & 0.4 & 1.1 & 31.917 \\
\hline
\end{tabular}

\section{Flame speed vs equivalence ratio}

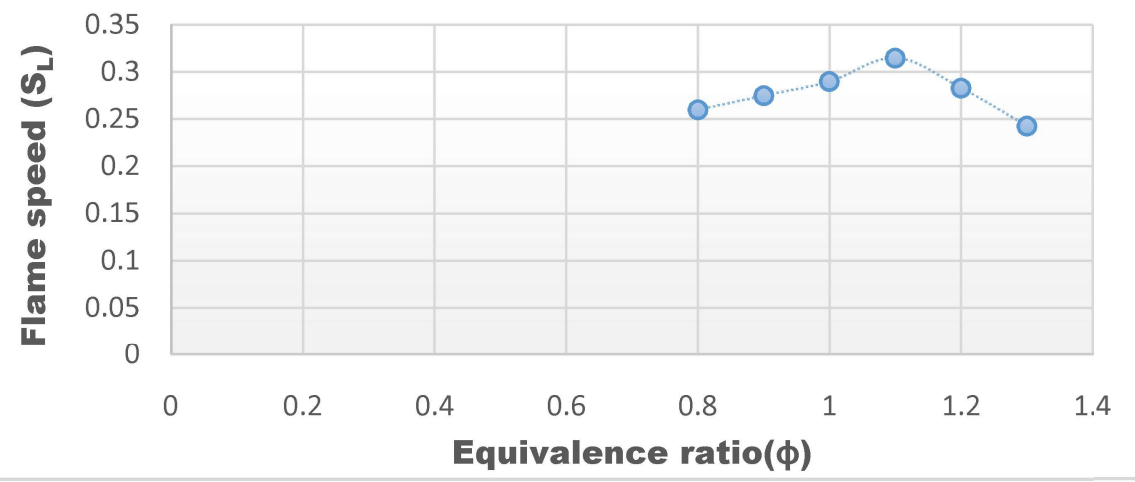

Fig. 4 Flame speed vs equivalence ratio 


\section{CONCLUSION}

1. From table 3 it is observed that flame propagation speed average $0.28 \mathrm{~m} / \mathrm{s}$. But in practical case flashback occur when Air-fuel $\left(\mathrm{CH}_{4}\right)$ velocity is higher than the observed value (i.e., $0.28 \mathrm{~m} / \mathrm{s}$ ). This may be due to disturbance of surrounding ambient air. So to avoid this error, it is better to protect flame by nitrogen or any other unreactive gas.

2. From images, it is noticed that for the same average unburnt mixture velocity, slightly rich $(\phi=1.1)$ condition shows a larger flame cone angle than all other condition i.e. stoichiometry, lean \& rich $(\phi$ $>1.1)$. That means at slightly rich $(\phi=1.1)$ condition, flame gives higher speed value.

3. It is realized that at the lean condition the flame structure is slightly lifted.

4. It may be noted that in some cases, depending on the mixture condition, the flame extinguishes suddenly without being lifted from the burner.

5. It is also noticed that flashback increases with the increase of fuel concentration.

\section{REFERENCE}

[1] Chen, J. L. P., \& Churchill, S. W. (1972). Stabilization of flames in refractory tubes. Combustion and Flame, 18(1), 37-42.

[2] Mishra, D. P. (2007). Experimental studies of flame stability limits of CNG-air premixed flame. Energy conversion and Management, 48(4), 1208-1211.

[3] Jejurkar, S. Y., \& Mishra, D. P. (2011). Flame stability studies in a hydrogen-air premixed flame annular microcombustor. International journal of hydrogen energy, 36(12), 7326-7338.

[4] Khandelwal, B., Sahota, G. P. S., \& Kumar, S. (2010). Investigations into the flame stability limits in a backward step micro scale combustor with premixed methane-air mixtures. Journal of Micromechanics and Microengineering, 20(9), 095030.

[5] Chen, Y. C., Chang, C. C., Pan, K. L., \& Yang, J. T. (1998). Flame lift-off and stabilization mechanisms of nonpremixed jet flames on a bluff-body burner. Combustion and flame, 115(1-2), 51-65.

[6] Leung, T., \& Wierzba, I. (2008). The effect of hydrogen addition on biogas non-premixed jet flame stability in a co-flowing air stream. International journal of hydrogen energy, 33(14), 38563862.

[7] Hu, S., Gao, J., Zhou, Y., Gong, C., Bai, X. S., Li, Z., \& Alden, M. (2017). Numerical and experimental study on laminar methane/air premixed flames at varying pressure. Energy Procedia, 105, 49704975.

[8] Harris ME, Grumer J, von Elbe G, Lewis B. Burning velocities, quenching and stability data of non-turbulent flames of methane, propane with oxygen and nitrogen. In: Proc 3rd Symp on Combustion, Flame and Explosion, Williams and Williams, Baltimore, 1949. pp. 80-8

[9] Wohl K, Kapp NM, Gazely C. Flame stabilization and quenching. In: Proc of 3rd Symp on Combustion, Flame and Explosion, Williams and Williams, Baltimore, 1949. pp. 3-20

[10] Schefer, R. W. (2003). Hydrogen enrichment for improved lean flame stability. International Journal of Hydrogen Energy, 28(10), 1131-1141.

[11] Jerzak, W., \& Kuźnia, M. (2016). Experimental study of impact of swirl number as well as oxygen and carbon dioxide content in natural gas combustion air on flame flashback and blowoff. Journal of Natural Gas Science and Engineering, 29, 46-54.

[12] Syred, N., Giles, A., Lewis, J., Abdulsada, M., Medina, A. V., Marsh, R., ... \& Griffiths, A. J. (2014). Effect of inlet and outlet configurations on blow-off and flashback with premixed combustion for methane and a high hydrogen content fuel in a generic swirl burner. Applied energy, 116, 288-296.

[13] Krikunova, A. I. (2020). Premixed methane-air flame under alternate gravity. Acta Astronautica.

[14] Yuasa, S., Oshimi, K., Nose, H., \& Tennichi, Y. (2005). Concept and combustion characteristics of ultra-micro combustors with premixed flame. Proceedings of the Combustion Institute, 30(2), 2455-2462.

[15] Mishra, D. P., \& Kannan, R. (2004). Experimental investigation of lean premixed swirl burner. International Journal of Turbo and Jet Engines, 21, 103-114. 